\title{
Study on Cutting Stock Optimization for Decayed Wood Board Based on Genetic Algorithm
}

\author{
Lin Wenshu, Mu Dan and Wu Jinzhuo*
}

College of Engineering and Technology, Northeast Forestry University, Harbin, Heilongjiang, 150040, P.R. China

\begin{abstract}
When making wood boards, the defects on the boards can reduce the strength of timber, and influence the machining process automation degree as well as the decoration quality or appearance after processing. Therefore, how to remove wood defects quickly and accurately and realize optimal cutting stock have always been a research hotspot in the field of wood processing. In this paper, based on the decayed wood board, the optimal scheme for cutting stock combination and mathematical model were designed, and the genetic algorithm that imitates the biological evolution was applied to code some optimization schemes initialized by chance. These schemes were improved by selection, crossover and mutation operation, and finally converged to the optimum. The results showed that genetic algorithm can achieve the cutting stock optimization for decayed wood boards. Through the realization of genetic algorithm in MATLAB, the wood board utilization rate reached $95.9 \%$, which greatly improved the utilization rate of wood.
\end{abstract}

Keywords: Cutting stock optimization, Defects, Genetic algorithm, Wood board.

\section{INTRODUCTION}

With the development of modern technology, the development and application of automatic system for automatic detection and recognition of sawn timber surface defects, edge cutting and cutting-off of sawn timber, and sawn timber grading is not only a hot topic in the current timber production industry, but also an important subject of study in timber processing industry for a long period in the future. With the combination of computer vision technology, image processing technology, and mathematical optimization model, the study on the detection of timber surface defects can be carried out and the optimized stock cutting plan can be determined, which will promote nondestructive detection of lumber and have important theoretical significance, promising application prospect and good economic and social benefits.

In this paper, the genetic algorithm which is a rapidly developing artificial intelligence technique was studied. Based on the information related to wood board and defects detected by computer vision recognition system, genetic algorithm was used to make decision on the optimal cutting stock scheme, which helped enable smooth operation of production lines, improve enterprise productivity and realize intelligent automatic control of wood board stock cutting. Such a plan can not only reduce workers' labor intensity, but also improve productivity and material utilization rate in timber processing industry, enabling sawmills to gain higher economic benefits during material processing. This study is of important significance in the development of intelligent

*Address correspondence to this author at the College of Engineering and Technology, Northeast Forestry University, Harbin, 150040, P.R. China; Tel: 15663790610; E-mail: wujinzhuo1980@163.com automation of timber processing and in relieving shortage of timber supply in China.

\section{OPTIMIZED CUTTING STOCK COMBINATION PLAN}

\subsection{Description of Stock Cutting Plan}

Since wood boards may have some kinds of defects, such as wormholes, decay, and knots, it is difficult to determine a rational wood board cutting stock plan. So far, there haven't been best optimization methods to solve the problem once for all. The current optimized wood board cutting stock plans based on genetic algorithm didn't take the defects on wood boards into consideration [1-5].

Therefore, with reference to domestic and foreign literatures related to optimal stock cutting problem, genetic algorithm is chosen in this paper to make optimization calculation for stock cutting of defective wood board. Since the positions of defects are fixed, their information can be obtained with the aid of computer vision technology such as industrial camera. The size of the defect is approximately rounded to simulate a small wood board size to be cut. In other words, the position of the defect is deemed as a fixed and unchangeable special board part to be cut. When MATLAB is used to run genetic algorithms for screening from generation to generation, the place of the defect is set to be 0 . When the place of the defect is considered during selection, the defect will be skipped automatically and it will not come to the choice of the next generation. The area of the wood board around the defect is divided into 4 regional blocks. For the convenience of programming and processing, the small board parts to be cut are assigned to numbers of an array. As shown in Fig. (1), the blocks of the wood board are 


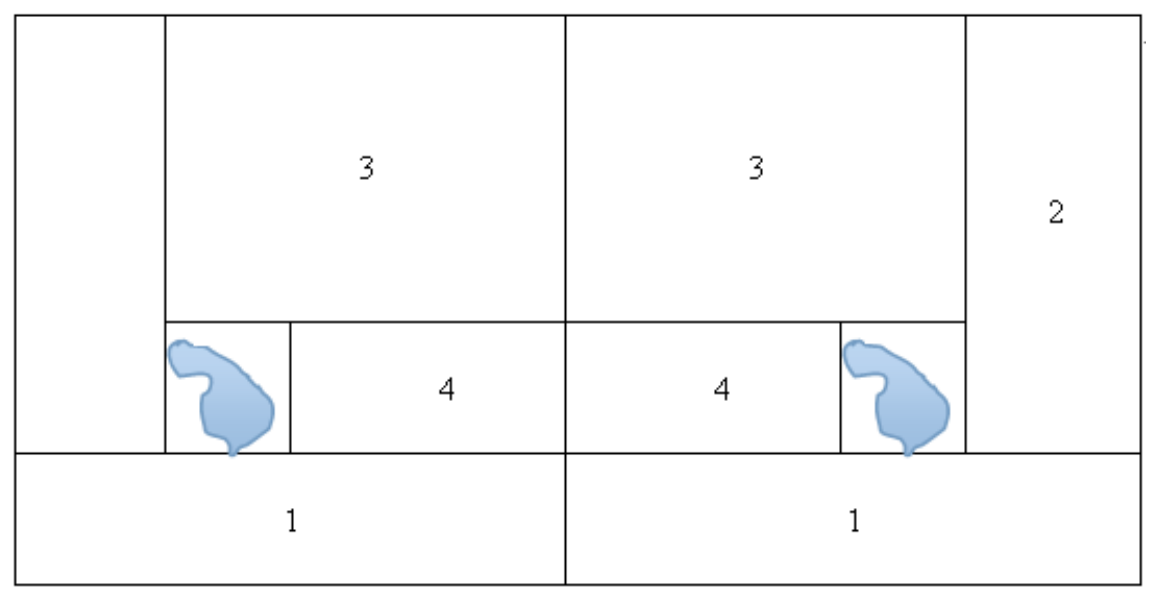

Fig. (1). Defected board cutting stock scheme.

defined as \#1 block, \#2 block, \#3 block, and \#4 block. The shaded part is the position of the defect. In this way, there aren't any defects in the four blocks. Then genetic algorithm is used to arrange nesting and cutting. Such plan will be considered on a wood board under a universal principle. This is a typical combinatorial optimization problem. When cutting is planned with the other three sides as the base respectively, the arrangements will be different. Therefore, a total of 10 stock cutting plans should be obtained.

\subsection{Mathematical Description of Stock Cutting Plan}

It is assumed that the length of a wood board is $L$, width is $K$ and area is $S$. For the small board part to be cut, its length is $x_{i}$, width is $y_{i}$ ( $i$ represents the $i^{\text {th }}$ kind of small board parts to be cut, $i=1,2,3 \ldots, n)$, and the area is $s_{i}$. The small board parts can be arranged horizontally or vertically. When the small board parts are arranged horizontally, the occupied space is $l_{i}$, and when they are arranged vertically, the occupied space is $k_{i} . x_{i} l_{i}$ represents the number of rows of small board parts when the parts are arranged horizontally in block $j(j=1,2,3,4) . y_{i} k_{i}$ represents the number of rows of small board parts when the parts are arranged vertically in block $j . n_{j}$ represents the number of small board parts in one row when they are arranged horizontally in block $j$, and $m_{j}$ represents the number of small board parts in one row when they are arranged vertically in block $j$ [6]. The defect is deemed as a small board part, and the four extreme coordinates of the defect are $x_{\text {min }}, x_{\text {max }}, y_{\text {min }}, y_{\max }$. For the case in Fig. (1), the objective function of the mathematical model is defined as:

$W_{\min }=S-\sum_{i=1}^{n} \sum_{j=1}^{4}\left(n_{j} x_{i} l_{i}+m_{j} y_{i} k_{i}\right) \times s_{i}$
It means the minimum total area of residual material in case of small board parts arranged on a wood board. Besides, the stock nesting must meet the following constraints:

(1) In the longitudinal direction of the wood board, the sum of the lengths of all small board parts should be less than or equal to $\mathrm{L}$.

$L-\sum_{j=1}^{4}\left(n_{j}+m_{j}\right) \geq 0$

(2) In the transversal direction of the wood board, the sum of the widths of all small board parts should be less than or equal to $K$.

$$
K-\sum_{i=1}^{n}\left(x_{i} l_{i}+y_{i} k_{i}\right) \geq 0
$$

(3) No small board parts shall overlap with any defects. The position constraints for various areas are as follows:

$$
\left\{\begin{array}{l}
y_{\text {min }}-\left(n_{1} x_{1} l_{1}+m_{1} y_{1} k_{1}\right) \geq 0 \\
x_{\text {min }}-\left(n_{2} x_{2} l_{2}+m_{2} y_{2} k_{2}\right) \geq 0 \\
m_{3} y_{3} k_{3}+n_{3} x_{3} l_{3}-y_{\text {max }} \geq 0 \\
n_{4} x_{4} l_{4}+m_{4} y_{4} k_{4}-x_{\text {max }} \geq 0
\end{array}\right.
$$

\section{MATHEMATICAL MODEL BASED ON GENETIC ALGORITHM}

Genetic algorithm comes from simulation of biological genetic and evolutionary processes in natural environment. It is an adaptive global optimization probability search algorithm. Genetic algorithm is able to find the global optimal solutions without being dependent on the model, which is robust in solving nonlinear problems, has high parallelism, high efficiency and other advantages. The flowchart of genetic algorithm is shown in Fig. (2). 


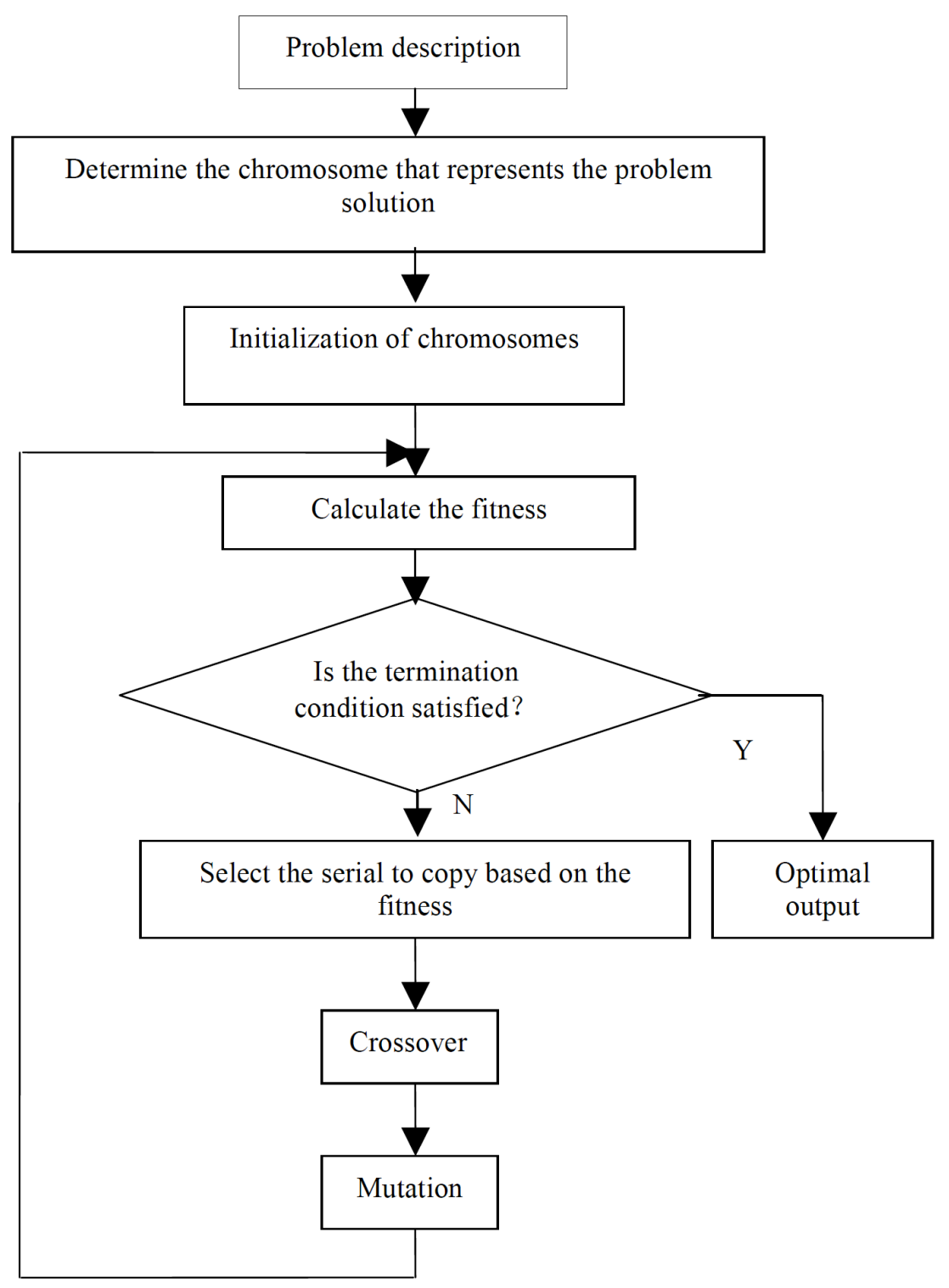

Fig. (2). Defected board cutting stock scheme.

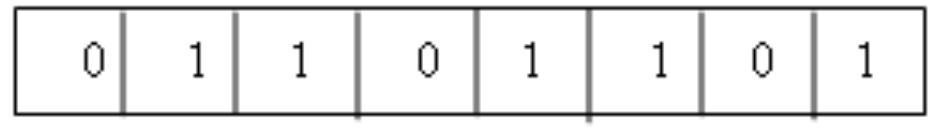

Fig. (3). The binary encoding patterns for chromosomes.

\subsection{Individual Chromosome Encoding}

In genetic algorithm, the feasible solutions of a practical optimization problem are converted to search space that can be handled by genetic algorithm. Such conversion method is called chromosome encoding. In genetic algorithm, the most critical step is chromosome encoding, which has great impact on mutation operation and mating chromosome. Binary representation encoding is used in this paper. Binary symbol series are used by chromosomes generated from binary encoding, with value 0 or 1 . The length of the selected binary coding chromosome is 20 . The binary encoding patterns for chromosomes are shown in Fig. (3).

\subsection{Population Initialization}

To set up an initial population, choose $\mathrm{N}$ as the size of the population. 20-digit binary number encoded chromosomes randomly generated are taken as the initial population. The rate of convergence in the genetic process can be affected 
Table 1. Board and defect size data.

\begin{tabular}{|c|c|c|c|c|c|c|c|c|c|}
\hline \multicolumn{2}{|c|}{ Board Size (cm) } & \multicolumn{2}{c|}{ Small Board Size 1 (cm) } & \multicolumn{2}{c|}{ Small Board Size 2 (cm) } & \multicolumn{2}{c|}{ X coordinates of the Defect } & \multicolumn{2}{c|}{ Y Coordinates of the Defect } \\
\hline \hline $\mathrm{L}(\mathrm{cm})$ & 12 & $\mathrm{x}_{1}(\mathrm{~cm})$ & 4 & $\mathrm{x}_{2}(\mathrm{~cm})$ & 3 & $\mathrm{x}_{\min }$ & 6 & $\mathrm{y}_{\min }$ & 4 \\
\hline $\mathrm{K}(\mathrm{cm})$ & 8 & $\mathrm{y}_{1}(\mathrm{~cm})$ & 2 & $\mathrm{y}_{2}(\mathrm{~cm})$ & 2 & $\mathrm{x}_{\max }$ & 8 & $\mathrm{y}_{\max }$ & 6 \\
\hline
\end{tabular}

Table 2. Selected parameters in genetic algorithm.

\begin{tabular}{|c|c|c|c|c|}
\hline Population Size & Length of Chromosome & Crossover Probability & Mutation Probability & Evolution Generation \\
\hline \hline 60 & 20 & 0.9 & 0.1 & 50 \\
\hline
\end{tabular}

by the size of the initial population. In order to get a satisfactory convergence effect, the size of the initial population must be properly chosen. In this paper, $\mathrm{N}$ takes a value of 60 .

\subsection{Determining the Value of Fitness Function}

Fitness function is an evaluation function for evaluating the merits of each chromosome and further for distinguishing their quality. The basis of evaluation is the objective function of the problem concerned, as shown in formula (5). In this paper, during fitness evaluation on individuals, if the fitness of some individual is zero, the algorithm flow can be terminated. The chromosome with zero fitness is the optimal solution. The optimal solution is then decoded to find out the desired optimized plan.

$$
f=S-\sum_{i=1}^{n} \sum_{j=1}^{4}\left(n_{j} x_{i} l_{i}+m_{j} y_{i} k_{i}\right) \times s_{i}
$$

\subsection{Crossover}

Crossover operation is to interchange the genetic characteristics of two parent individuals at a crossing point. Then two new individuals are generated. Such operation is very important in acquiring high-quality new individuals in genetic algorithm [6]. The detailed process is as follows: For each chromosome, if random $(0,1)$ is less than crossover probability $P_{c}$, it means such chromosome is suitable for crossover operation. Otherwise, the chromosome will be copied directly to the new population without any crossover operation. Random $(0,1)$ is an evenly distributed random number generator in the range of $[0,1]$. The method adopted in this paper is single-point crossover, and the specific operation is: a crossover point is set up randomly in the string of an individual chromosome. To perform crossover, just interchange the two genetic structures before and after the point. Then two new individuals are generated.

\subsection{Mutation}

Chromosome mutation is achieved through genetic process. With regard to each gene of the new population formed after crossover, judgment is made as to whether such gene can be mutated or not based on mutation probability $P_{m}$. If random $(0,1)$ is less than $P_{m}$, change the value of the gene. Otherwise, no mutation happens to the gene, and its value remains unchanged. To ensure the smooth operation of genetic algorithm, the probability of mutation should be set within an appropriate range.

\subsection{Termination Condition}

There are two types of common termination conditions for genetic algorithms. One is to set the maximum (genetic) number of generations. The other is to make judgment based on individual difference to take control through calculation of genetic diversity measurement or the similarity of all genes in a population. The first method is adopted by this paper and the maximum evolution generation is set to be 50 .

\section{EXAMPLE OF OPTIMIZED WOOD BOARD STOCK CUTTING}

The size of the wood board and the size of the required small boards, as well as the coordinates of the wood board defects are shown in Table $\mathbf{1}$. The parameters selected by genetic algorithm are shown in Table 2 . The prepared genetic algorithm codes are input into the software of MATLAB and the running results are shown in Fig. (4). In Fig. (4a), the horizontal axis represents the number of evolution generations, and the vertical axis represents the area of residual material. With continuous iterations or evolutions, the result tends to become stabilized to equilibrium. The final area of residual material is $4 \mathrm{~cm}^{2}$. Since the initial wood board area is $96 \mathrm{~cm}^{2}$, the rate of utilization of the wood board after optimized combination is $95.9 \%$, which meets the requirements of optimized wood board stock cutting. Fig. (4b) shows the chromosome binary code of the optimal solution, where the horizontal axis represents the length of chromosome, and the vertical axis represents the binary number. Therefore, the chromosome binary code of the optimal solution is 00100000010010000100 .

The optimized cutting arrangement of the board is shown in Fig. (5), where the shaded part represents the position and size of the defect. The result of the cutting consists of $5 \mathrm{spec}$. \#1 small board parts (4 arranged horizontally and 1 arranged vertically) and 8 spec. \#2 small board parts (4 arranged horizontally and 4 arranged vertically). The remaining area is the area of residual material. 


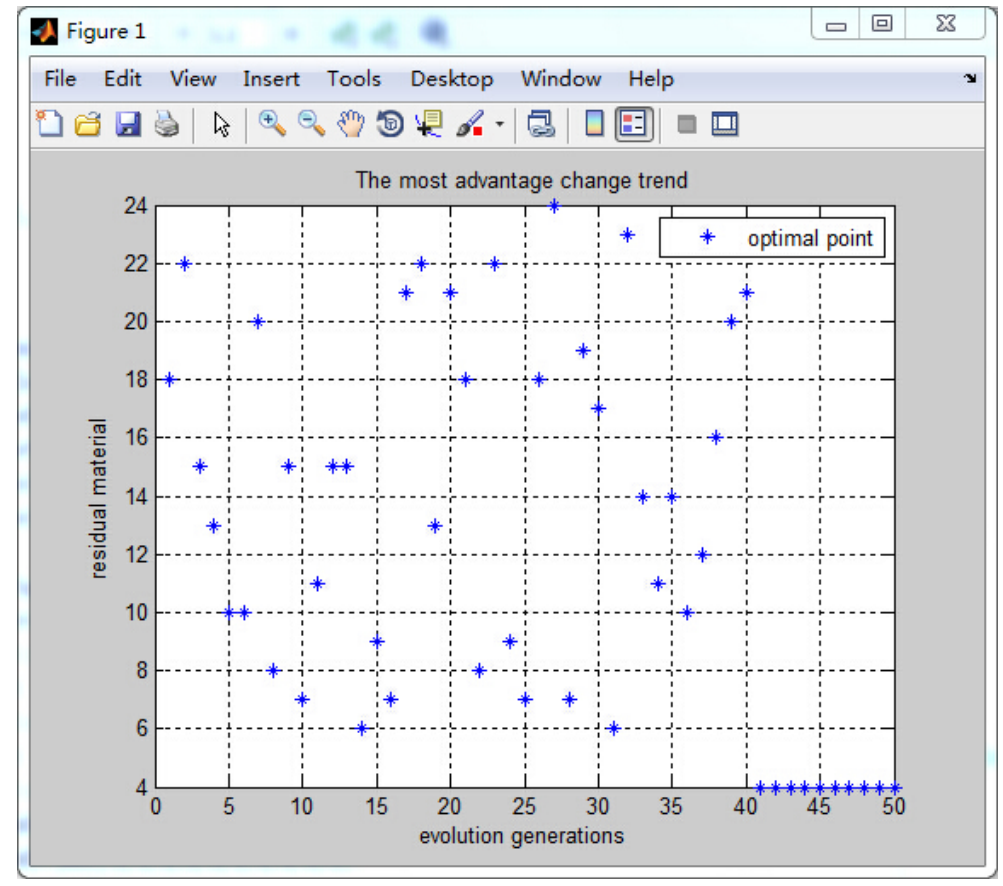

(a)

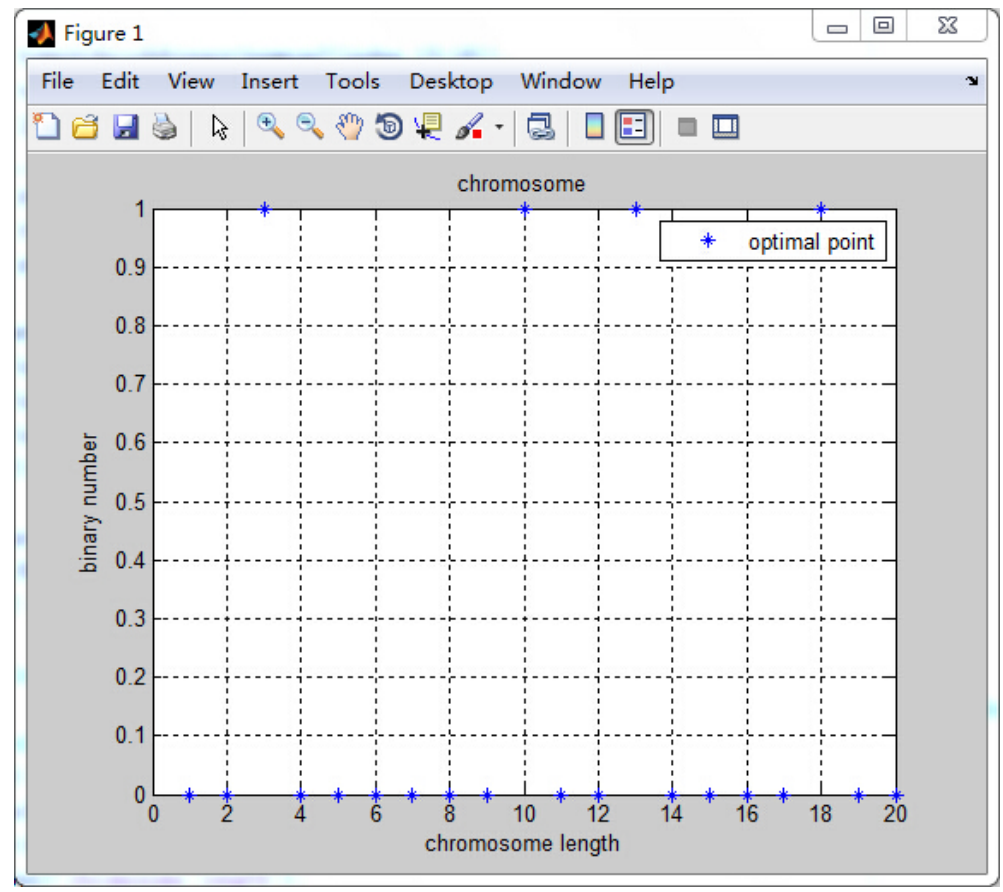

(b)

Fig. (4). Running results. (a) The most advantageous change trend. (b) The length of chromosome.

\section{CONCLUSION}

Genetic algorithm, as an artificial intelligence technology, is used in this paper to study the cutting plan of defective wood boards. An optimal mathematical model for board cutting is established with the objective of minimizing the residual material. The genetic algorithm was run with MATLAB. The optimal wood board cutting plan is finally determined through optimization treatment by selection, crossover and mutation. The result of operation indicated that genetic algorithm is able to solve optimal combination of board cutting problem. The utilization rate of defective wood board reached $95.9 \%$ through running genetic algorithm with MATLAB, which is significantly improved compared to other methods. In addition, the study results will play an active role in improving the automation level of defective wood board cutting. 


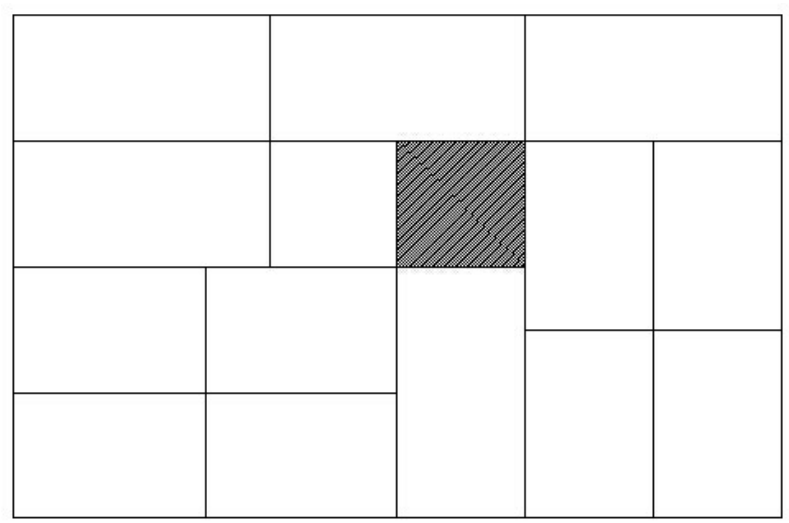

Fig. (5). Optimized cutting arrangement of board.

\section{CONFLICT OF INTEREST}

The authors confirm that this article content has no conflict of interest.

\section{ACKNOWLEDGEMENTS}

The authors would like to acknowledge the support of the Fundamental Research Funds for the Central Universities (DL13BB19) and the Scientific Activities Support Program of Chinese State Forestry Administration for Returned Talents from Overseas (415003).
[2] J. Sun, "Study and application of genetic algorithms on twodimensional cutting stock", M.S. thesis, Northeast Forestry University, Harbin, Heilongjiang, 2002.

[3] C.Z. Xing, and Y.Q. Sun, "Optimal board cutting based on simulated annealing genetic algorithm", Journal of Liaoning Technical University (Nature Science Edition), vol. 25, pp. 406-408, 2006.

[4] Y.P. Zhou, and Z.Y. Ti, "Application of genetic algorithm in combination optimization", Journal of Liaoning Technical University (Nature Science Edition), vol. 24, S1, pp. 283-285, 2005.

[5] Y.G. Chen, "The application of genetic algorithm and fuzzy control theory in control system of wood optimization cut", M.S. thesis, Beijing Forestry University, Beijing, 2003.

[6] J. Cao, and S. Feng, "The application of genetic algorithms in rectangular object optimal layout", Computer Engineering and Applications, vol. 5, pp. 5-8, 1999.

\section{REFERENCES}

[1] J. Cao, Q. Yue, Z.Y. Zhang, and K.L. Hu, "The utilization of genetic neural network algorithm on furniture optimal allocating problems", Forest Engineering, vol. 19, pp. 36-37, 2003.

(C) Wenshu et al.; Licensee Bentham Open.

This is an open access article licensed under the terms of the Creative Commons Attribution Non-Commercial License (http://creativecommons.org/licenses/by$\mathrm{nc} / 4.0 /$ ) which permits unrestricted, non-commercial use, distribution and reproduction in any medium, provided the work is properly cited. 